\title{
§ VI Diagnostic Medical Omens Concerned with Sick Eyes (Diagnostic Handbook, Sakikkû Chapter 5)
}

Manuscripts transliterated with bound text by Eric Schmidtchen, translated and edited by Strahil V. Panayotov.

TITLE šumma marșu in imittišu ikkalšu qāt il abǐš? ... imarrașma? iballuṭ If the sick man's right eye hurts him: (the cause is) the hand-of-hisfather's-god; he (the patient) will be sick (for x-days), but should recover.

\section{Manuscripts}

\begin{tabular}{|c|c|c|c|c|}
\hline $\mathrm{A}$ & K 6629 & AMT 75/2 & TDP I, 44-46 & 1'-16' \\
\hline B & BM 121063 & $\begin{array}{l}\text { forthcoming copy } \\
\text { by Schmidtchen }\end{array}$ & $\begin{array}{l}\text { - mentioned in Heeßel } \\
\text { 2000: } 133 \mathrm{fn} .20 .\end{array}$ & $\begin{array}{l}17^{\prime}-31^{\prime} \\
56^{\prime}-65^{\prime}\end{array}$ \\
\hline $\mathrm{C}$ & K 6737 & CT $37 / 50$ & TDP I, 46-48 & $\begin{array}{l}\text { 32'-41' } \\
70^{\prime}-78^{\prime} \\
(B A D \text { 49'- } \\
\left.60^{\prime}\right)\end{array}$ \\
\hline $\mathrm{D}$ & K 13985 & TDP II, Pl. IX & TDP I, 46-48 & $\begin{array}{l}\text { 42'-49', } \\
\text { 79'-84' } \\
(B A D \text { 61'- } \\
\left.67^{\prime}\right)\end{array}$ \\
\hline $\mathrm{E}$ & K 10570 & TDP II, Pl. IX & TDP I, 48-50 & $\begin{array}{l}50^{\prime}-56^{\prime} \\
87^{\prime}-98^{\prime} \\
(B A D \text { 68'- } \\
\left.80^{\prime}\right)\end{array}$ \\
\hline $\mathrm{F}$ & K 8078 & - & - & 57'-69' \\
\hline G & W 17360g & TDP II, Pl. LXVI & TDP I, 48 & $\begin{array}{l}\text { 79'-86' } \\
(B A D \text { 61'- } \\
\left.67^{\prime}\right)\end{array}$ \\
\hline $\mathrm{H}$ & $\begin{array}{l}\text { K } 3957+\text { K } 6347 \\
+ \text { K } 9350\end{array}$ & AMT $105 / 2$ & TDP I, 50-54 & $\begin{array}{l}\text { 99'-112' } \\
\left(B A D 81^{\prime}-\right. \\
\left.99^{\prime}\right)\end{array}$ \\
\hline I & K 7099 & СТ $37 / 50$ & TDP I, 50-52 & $\begin{array}{l}\text { 113'-135' } \\
\left(B A D 100^{\prime}-\right. \\
\left.126^{\prime}\right)\end{array}$ \\
\hline
\end{tabular}




\begin{tabular}{|l|l|l|l|l|}
\hline J & K 10691 & TDP II, Pl. XVIII & $\begin{array}{l}\text { - uncertain placement, } \\
\text { but see Schmidtchen, } \\
\text { forthcoming, Mesopota- } \\
\text { mische Diagnostik, Tafel }\end{array}$ & \\
& & & $\begin{array}{l}\text { 132'(?) } \\
5\end{array}$ \\
\hline
\end{tabular}

\section{Commentary}

\begin{tabular}{|l|l|l|l|l|}
\hline a & W 22307/16 & SpTU I, Nr. 31 & SpTU I, 39-40; & 1, 11', 14'(?), \\
& & Clancier 2009: 480; & 52', 56'- \\
& & & 57 '(?), 58'- \\
& & & & 59 '(?), \\
& & & & 100 '(?), \\
& & & & $104(?), 107^{\prime}$, \\
& & & & 108 ', 120' \\
\hline
\end{tabular}

NB.

For an eclectic English edition of tablet 5, without variants, but with concise explanatory notes, compare Scurlock 2014: 45ff.

\section{Edition}

\begin{tabular}{|c|c|}
\hline BREAK OF UNKNOWN LENGTH! & BREAK OF UNKNOWN LENGTH! \\
\hline $\begin{array}{l}\text { 1' } \quad[\text { šumma] ìn šumēlišu? [...] amēlu } \\
\text { šü? }[. . .]\end{array}$ & $\begin{array}{l}{ }^{\prime}[\text { Iff }] \text { his left eye }[\ldots] \text { this man will? }[\ldots] \\
{ }^{2}[\text { If }] \text { his right eye is injured and }[. . .]\end{array}$ \\
\hline 2' [šumma] īn imittišu pa'șatma [...] & ${ }^{3}[$ If] his left eye is injured and [...] \\
\hline 3' [šumma] ìn šumēlišu pa'șatma [...] & ${ }^{4}[$ If $]$ his both eyes are injured and [...] \\
\hline 4' [šumma] ìnāšu pa'șāma [...] & ${ }^{5}[$ [If] his right eye is injured and [...] \\
\hline 5' [šumma] īn imittišu pa'șatma [...] & ${ }^{6}$ If his left eye is injured and [...] \\
\hline 6' šumma ìn šumēlišu pa'șatma [...] & ${ }^{7}$ If his both eyes are injured and [discharge] \\
\hline $\begin{array}{l}\text { 7’ šumma ìnāšu pa'șāma kalâ? [nadâ } \\
\text {...] }\end{array}$ & $\begin{array}{l}\text { yellowish kalû-secretion [...] } \\
{ }^{8} \text { If his right eye discharges yellowish kalû- }\end{array}$ \\
\hline 8' šumma ìn imittišu kalâ nadât [...] & $\begin{array}{l}\text { secretion [...] } \\
\text { 9'If his left eye discharges yellowish kalû- }\end{array}$ \\
\hline 9' šumma ìn šumēlišu kalâ nadât [...] & $\begin{array}{l}\text { secretion }[. . .] \\
{ }^{10} \text { 'If his both eyes discharge yellowish kalû- }\end{array}$ \\
\hline 10’ šumma ìnāšu kalâ nadâ [...] & $\begin{array}{l}\text { secretion }[. . .] \\
{ }^{11} \text { If his right eye is dark }[\ldots]\end{array}$ \\
\hline 11' šumma ìn imittišu tarkat [...] & $\begin{array}{l}{ }^{12} \text { If his left eye is dark: it will be prolonged } \\
\text { and [...] }\end{array}$ \\
\hline
\end{tabular}




\begin{tabular}{|c|c|}
\hline $12^{\prime}$ & šumma ìn šumēlišu tarkat irrikma \\
\hline & {$[\ldots]$} \\
\hline 13' & šumma īnāšu tarkā irrikma [...] \\
\hline 14' & $\begin{array}{l}\text { šumma ìn imittišu tarkatma lāa } \\
\text { ipettē ... [...] }\end{array}$ \\
\hline $15^{\prime}$ & $\begin{array}{l}\text { [šumma ìn šumēlišu] tarkatma lā } \\
\text { ipettēe }[. . .]\end{array}$ \\
\hline 16 ' & $\begin{array}{l}\text { [šumma ìnāšu tarkāma] lā ipettē } \\
{[\ldots]}\end{array}$ \\
\hline & EAK OF UNKNOWN LENGTH! \\
\hline
\end{tabular}

17' [šumma ...] iballut

18' ...

19' [šumma ...] iballut?

20' [šumma ...] iballut

21' [šumma ... qāt] ilišu?

22' [šumma ...] iballut

23' [šumma ... qāt] il ālišu

24' [šumma .... iballut?

25' [šumma ... murussu] ikabbit?

26' [šumma ... murussu] ikabbit?

27’ [šumma ...] imât

28' [šumma ...] irrikma? iballut

29' [šumma ...] irrikma? iballut?

30' [šumma ...] imât?

31' ...

\section{BREAK OF UNKNOWN LENGTH!}

32' [šumma ...] imât

33' [šumma ...] imât

34' [šumma ... murussu?] irrik

35' [šumma ...] imât

36' [šumma ... murussu'] ikabbitma imât

37’ [šumma ... murussu?] irrikma na'id

38' [šumma ... murussu] irrikma iballut

39' [šumma ...] imât

40' [šumma ...] imât

41' [šumma ...] hìța ul ǐšū

BREAK OF UNKNOWN LENGTH!

42' [šumma ... qāt] Ištar
${ }^{13}$ 'If his both eyes are dark: it will be prolonged and [...]

${ }^{14}$ If his right eye is dark and he cannot open (it) ... [...]

${ }^{15}$ 'If his left eye[ is dark and he cannot open [(it) ...]

${ }^{16}$ [If his both eyes are dark and he] cannot open (them) [...]

\section{BREAK OF UNKNOWN LENGTH!}

${ }^{17}$ '[If ...] he should recover.

${ }^{18}$ '...

${ }^{19}$ '[If ....] he should recover.?

${ }^{20}$ '[If ...] he should recover.

${ }^{21}$ '[If ... (the cause is) the hand]-of-his god.

${ }^{22}$ '[If ...] he should recover.

${ }^{23}$ '[If ... (the cause is) the hand]-of-his city god.

${ }^{24}$ [If ...] he should recover.?

${ }^{25}$ [If ... his sickness] will be grave?

${ }^{26}$ [If ... his sickness] will be grave.

${ }^{27}$ [If ...] he will die.

${ }^{28}$ '[If ...] it will be prolonged and he should recover.

${ }^{29}$ [If ...] it will be prolonged? and he should recover. ${ }^{30}$ '[If ...] he will die. ${ }^{31}$ '...

\section{BREAK OF UNKNOWN LENGTH!}

${ }^{32}$ [If ...] he will die.

${ }^{33}$ 'If .... he will die.

${ }^{34}$ [If ... his sickness?] will be prolonged.

${ }^{35}$ [If ...] he will die.

${ }^{36}$ [If ... his sickness?'] will be grave and he will die.

${ }^{37}$ [If ... his sickness?'] will be prolonged and he/it is worried/worrying.

${ }^{38}$ '[If ... his sickness] will be prolonged but he should recover.

${ }^{39}$ 'If ...] he will die.

${ }^{40}$ '[If ...] he will die. ${ }^{41}$ [If ...] there is no fault.

BREAK OF UNKNOWN LENGTH!

${ }^{42}$ [If ... (the cause is) hand]-of-Ištar. 


\begin{tabular}{|c|c|}
\hline 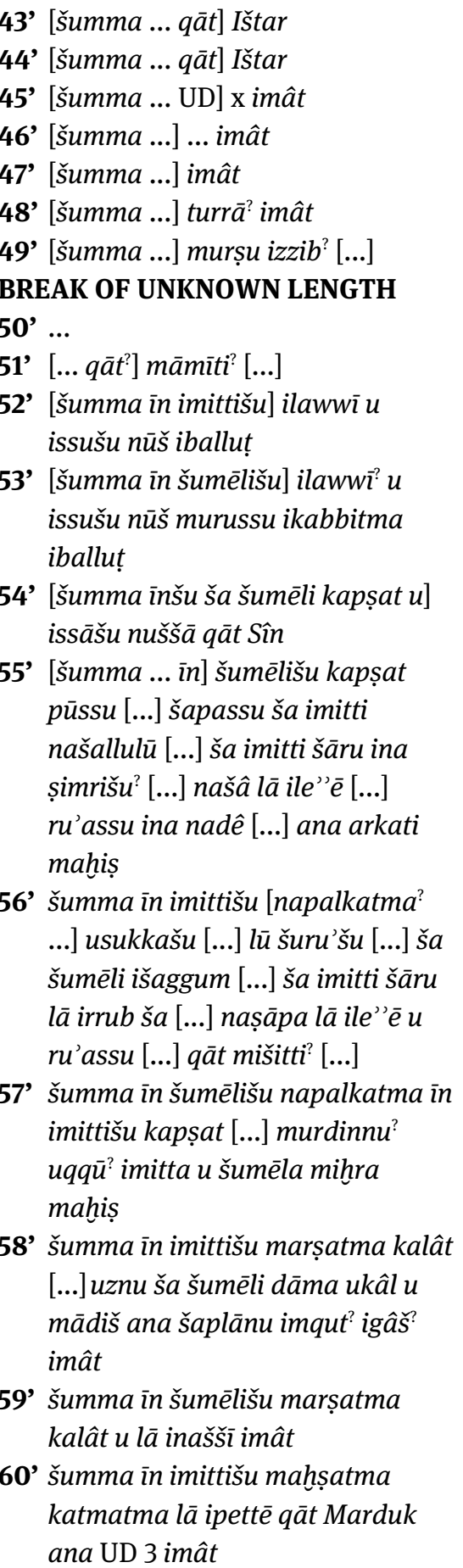 & 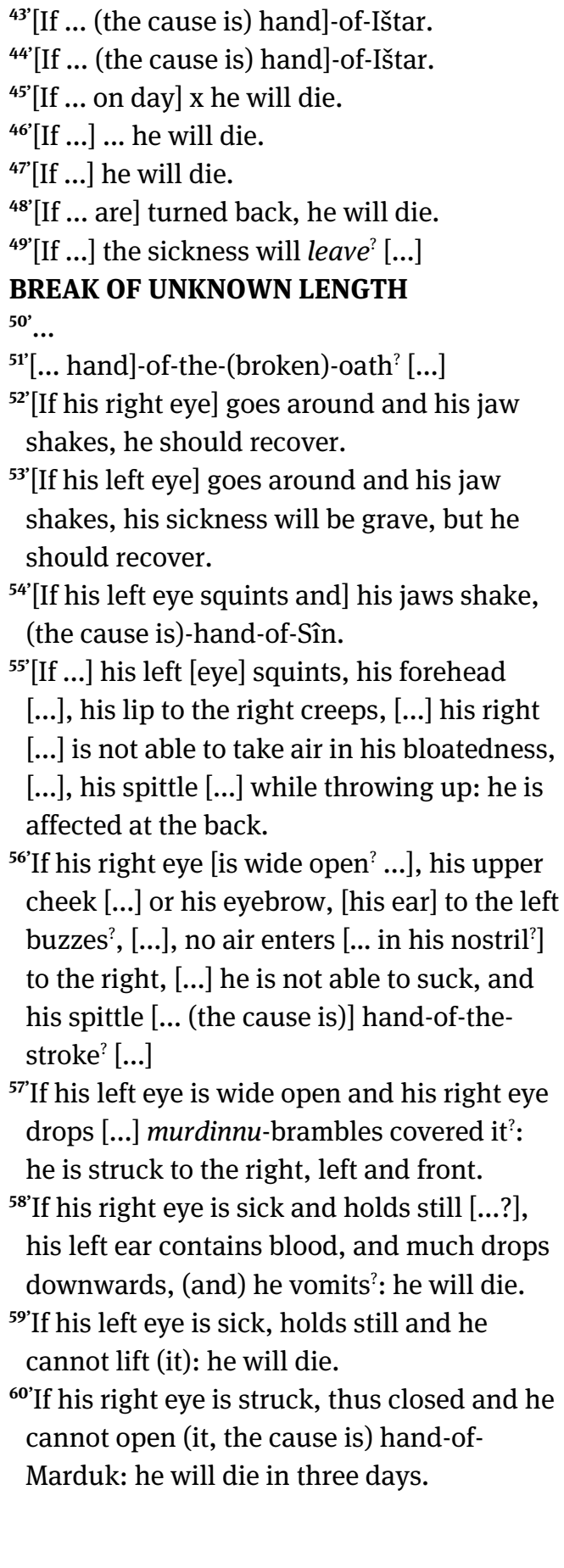 \\
\hline
\end{tabular}


61' šumma in šumēlišu mahșatma katmatma lā ipettē qāt Adad ana UD 4 imât

62' šumma ìn šumēlišu mahșatma kalât te'ât u lā inatțal imât

63’ šumma ìn šumēlišu? katmat irrikma imât?

64' [šumma ìn imittišu/šumēlišu?] katmatma lā ipettē [...]

65'...

66' [šumma...] qāt [...]

67' [šumma...] iraššì qāt [...]

68' [šumma...] ìtenezzebšu? ikașșaș? [...] $q \bar{a} t[. .$.

69' [šumma...] qāt [...]

\section{BREAK OF UNKNOWN LENGTH}

70'...

71’ šumma ìnāšu qadūta [sāmta malâ ...]

72’ šumma ìnāšu qadūta [sāmta malâ ...]

73’ šumma ìnāšu qadūta sāmta [malâ ...] / marșu šū iballuṭ [...]

74' šumma ìnāšu dāma malâ [imât']

75' šumma ìnāšu dāma malâ [...]

76’ šumma ìnāšu dāma malâ [...]

77’ šumma ìnāšu dāma malâ [...] šibit šāri? [...] 78'šumma īnāšu [...]

BREAK OF UNKNOWN LENGTH

79’ šumma [īnāšu ...]

80’ šumma īnāšu kīma? [...]

81' šumma ìnāšu kīma? [...]

82' šumma ìnāšu ibakkâ [...]

83' šumma ìnāšu ibakkâ birdu? [...]

84' šumma ìnāšu dimāti irtanaššâ [...]

85' [šumma ìnāšu] dimāti ittanaddâ

$\mathbf{8 6}^{[\ldots]}$

BREAK OF UNKNOWN LENGTH

87’ šumma īnāšu [...]

88' šumma īnāšu [...] marșu šü? [...]

89’ šumma ìnāšu balṣa $\bar{a}^{\text {[...] }}$
${ }^{61}$ 'If his left eye is struck, thus closed and he cannot open (it, the cause is) hand-of-Adad: he will die in four days.

${ }^{62}$ 'If his left eye is struck, holds still, smothered and he cannot see: he will die.

${ }^{63}$ 'If his left eye is closed, (the sickness) will be prolonged and he will die.

${ }^{64}$ '[If his right/left eye] is closed and he cannot open [(it) ...]

65....

${ }^{66}$ '[If ... (the cause is)] hand-of-[...]

${ }^{67}$ [If ...], he has (... the cause is) hand-of-[...]

${ }^{68}$ '[If ... it? ${ }^{\text {? }}$ always abandons him, he gnashes (his teeth) [... (the cause is)] hand-of-[...] ${ }^{69}$ '[If ... (the cause is)] hand-of-[...]

\section{BREAK OF UNKNOWN LENGTH} ${ }^{70}$ '...

${ }^{71}$ If his eyes [are full of red] silt [...]

${ }^{72}$ If his eyes [are full of red] silt [...]

${ }^{73}$ 'If his eyes [are full of] red silt [...] / this sick man should recover. [...]

${ }^{74}$ If his eyes are full of blood: [he will die?].

${ }^{75}$ 'If his eyes are full of blood: [...]

${ }^{76}$ 'If his eyes are full of blood: [...]

${ }^{77}$ If his eyes are full of blood: [... (the cause is)] wind-blast ${ }^{?}[. .$.

${ }^{78}$ 'If his eyes [...]

\section{BREAK OF UNKNOWN LENGTH}

${ }^{79}$ ' If his [eyes ...]

${ }^{80}$ If his eyes are like [...]

${ }^{81}$ 'If his eyes are like [...]

${ }^{\mathbf{8 2}}$ 'If his eyes weep [...]

${ }^{83}$ 'If his eyes weep, (having) pockmark [...]

${ }^{84}$ If his eyes have tears [...]

${ }^{85}$ 'If his eyes] are constantly giving off tears [...]

86'...

\section{BREAK OF UNKNOWN LENGTH}

${ }^{87}$ 'If his eyes are [...]

${ }^{88}$ 'If his eyes are [...] this sick-man [...]

${ }^{89}$ 'If his eyes are staring [...] 


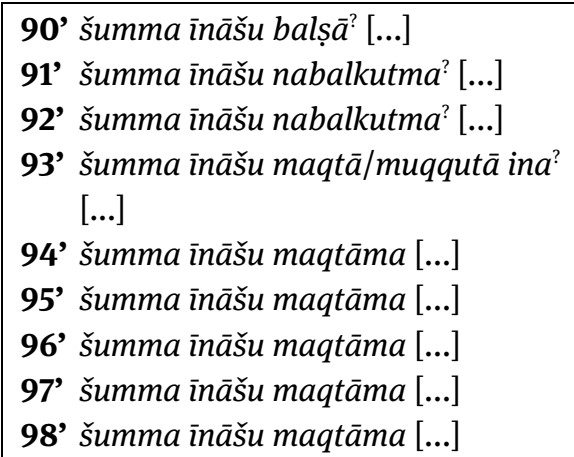

BREAK OF UNKNOWN LENGTH

99' [šumma] ìnāšu [...]

100’ šumma innāšu [(burkāšu kirrāšu ... mithāriš ikkalāšu? )] ištū [murussu ittabšû ana aganutillê] itâršumma [ina ūm tìk šamê imât']

101' šumma ìnāšu [itteneprikk $\bar{a}^{\text {? ....] }}$

102' šumma ìnāšu [itteneprikka $\bar{a}^{\text {....] }}$

103’ šumma īnāšu [lapit/ulappat? ...]

104’ šumma ìnāšu ișșanundā [...] u minâtūšu illakă $\bar{a}^{?}[. .$.

105’ šumma īnāšu ișșanundā u [...]

106' šumma ìnāšu izzakâ/izzakkâ? ... [...]

107’ šumma īnāšu u'ammaș [... dāmū?] / ina pî̌su illakū [...]

108’ šumma ìnāšu bērā [...]

109’ šumma ìnāšu kabtāma [...]

110’ šumma ìnāšu elī [...]

111’ šumma ìnāšu ... [...]

112’ šumma īnāšu ... [...]

\section{BREAK OF UNKNOWN LENGTH}

113' [šumma pūṣ? īnīšu qû?'] arqūtu iprikü? $[. .$.

114' [šumma pūṣ? ìnišu] urqa balil [...]

115' [šumma pūṣ? īnīšu] nabalkutma [...]

116’ [šumma pūṣ ìnišu] nabalkutma elēnu u [šaplānu ...]

117’ šumma pūṣ īnī̌u nabalkutma šēpāšu kașâ [...]
${ }^{90}$ 'If his eyes are staring [...]

${ }^{91}$ If his eyes are twisted and [...]

${ }^{92}$ 'If his eyes are twisted and [...]

${ }^{93}$ 'If his eyes are sunken, in? [...]

${ }^{94}$ 'If his eyes are sunken, [...]

${ }^{95}$ If his eyes are sunken and [...]

${ }^{96}$ If his eyes are sunken and [...]

${ }^{97}$ If his eyes are sunken and [...]

${ }^{98}$ If his eyes are sunken and [...]

\section{BREAK OF UNKNOWN LENGTH}

${ }^{99}$ [If] his eyes [...]

${ }^{100}$ 'If his eyes, [(his knee, his collarbone and

... hurt him altogether?)], since [his sickness appeared] and changed [into dropsy] then [he will die on a rainy day.]

${ }^{101}$ 'If his eyes [constantly malfunction ...]

${ }^{102}$ 'If his eyes [constantly malfunction ...]

${ }^{103}$ 'If he [touches] his eyes [...]

${ }^{104}$ 'If his eyes circle around [...] and his limbs move [...]

${ }^{105}$ 'If his eyes circle around [...]

${ }^{106}$ 'If his eyes are clear? [...]

${ }^{107}$ 'If he flays his eyes [... blood?] / flows out of his mouth [...]

${ }^{108}$ 'If his eyes are barely opened [...]

${ }^{109}$ 'If his eyes are heavy [...]

${ }^{110}$ 'If his eyes are over [...]

${ }^{111}$ 'If his eyes are ... [...]

${ }^{112}$ 'If his eyes are ... [...]

\section{BREAK OF UNKNOWN LENGTH}

${ }^{113}$ '[If] yellow [filament] covers [the white of his eyes ....

${ }^{114}$ [If the white of his eyes] is mixed with yellow colour [...]

${ }^{115}$ '[If the (white) of his eyes] is rolled up [...] ${ }^{116}$ ' [If the (white) of his eyes] is rolled up from above [and below ...]

${ }^{117}$ '[If the white of his eyes] is rolled up and his feet cold [...] 
118’ šumma pūṣ īnī̌ru nabalkutma šēpāšu tebâa? [...]

119’ šumma ṣulum īnišu nabalkutma [...]

120’ šumma șulum īnišu nabalkutma šēpāšu ana libbišu [turrāa? ...]

121' šumma šer'ānū ìnīš kìma unqi kappū [...] nakid [...]

122’ šumma šer'ānū ìnišu kìma šitê [... lū] qāt ilišu lū qāt il ālišu [...]

123’ šumma šer'ānū ìnišu illakūma ša nakkapti šaknū [...]

124' šumma šer'ānū kakkulti ìn imittišu șalmū [...]

125’ šumma šer'ānū kakkulti ìn šumēlišu șalmū [...]

126’ šumma šer'ānū kakkulti ìnīšu șalmū [...]

127' šumma šer' ānū kakkulti ìn imittišu tarkū [...]

128' šumma šer'ānū kakkulti ìn šumēlišu tarkū [...]

129' šumma šer'ānū kakkulti ìn imittišu [du' umü? ...]

130’ šumma šer' ānū kakkulti īn šumēlišu [du'umū? ...]

131’ šumma šer'ānū kakkulti īnišš [du"umū?...]

132’ šumma kibrāt īnī̌u naphā [...] naphū [...]

133’ šumma kappū īnī̌̌ šinnâ? tiṣbutū [...]

134’ šumma kappū īnī̌̌ ša imitti [...] tiṣbutū? u ummu șarhu [...] qāt Sîn? : qāt Šamaš

135’ šumma [...] īnī̌s [...] qû? iprikū? qāt Sîn : qāt Šamaš

136' [šumma... iniňš ...] šer'ānu? arqu iprik qāt Sîn : qāt Šamaš

137' [šumma...] qāt Ninurta
${ }^{118}$ [If the white of his eyes] is rolled up and his feet throb [...]

${ }^{119}$ 'If the pupil (lit. 'black') of his eyes is rolled up, then [...]

${ }^{\mathbf{1 2 0}}$ If the pupil (lit. 'black') of his eyes is rolled up and his feet are [turned] towards his belly [...]

${ }^{121}$ 'If the blood vessels of his eyes are bowed like a ring [...]: he is in a critical state [...]

${ }^{122}$ 'If the blood vessels of his eyes are [...] like a textile [... or ... (the cause is)] hand-of-hisgod or hand-of-his-city [...]

${ }^{123}$ 'If the blood vessels of his eyes are moving, but those on his temple are still [...]

${ }^{24}$ If the blood vessels of his right eyeball are black [...]

${ }^{125}$ If the blood vessels of his left eyeball are black [...]

${ }^{126}$ 'If the blood vessels of his both his eyeballs are black [...]

${ }^{127}$ 'If the blood vessels of his right eyeball are dark [...]

${ }^{128}$ 'If the blood vessels of his left eyeball are dark [...]

${ }^{129}$ 'If the blood vessels of his right eyeball are [dark-red ...]

${ }^{130}$ 'If the blood vessels of his left eyeball are [(dark-red?) ...]

${ }^{131}$ 'If the blood vessels of his both eyeballs are [(dark-red?) ...]

${ }^{132}$ 'If the corners of his both eyes are swollen [...] swollen [...]

${ }^{133}$ 'If the lids of his both eyes grasp each other [...]

${ }^{134}$ 'If the lids of his right eye [...] the grasp and flaring up ummu-fever [...] hand-of-Sîn : hand-of-Šamaš.

${ }^{135}$ 'If [...] of his eyes [... and] filaments cover: hand-of-Sîn : hand-of-Šamaš.

${ }^{136}$ [If ... his eyes ...] yellow colour covers the blood vessels: hand-of-Sîn : hand-of-Šamaš. ${ }^{137}$ '[If ...] hand-of-Ninurta. 


\begin{tabular}{|c|c|}
\hline 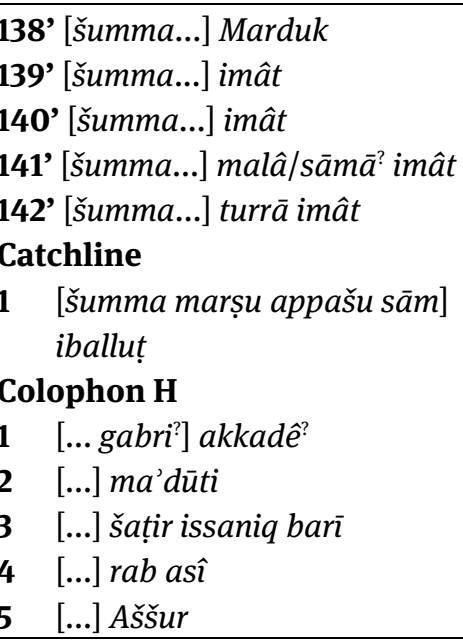 & 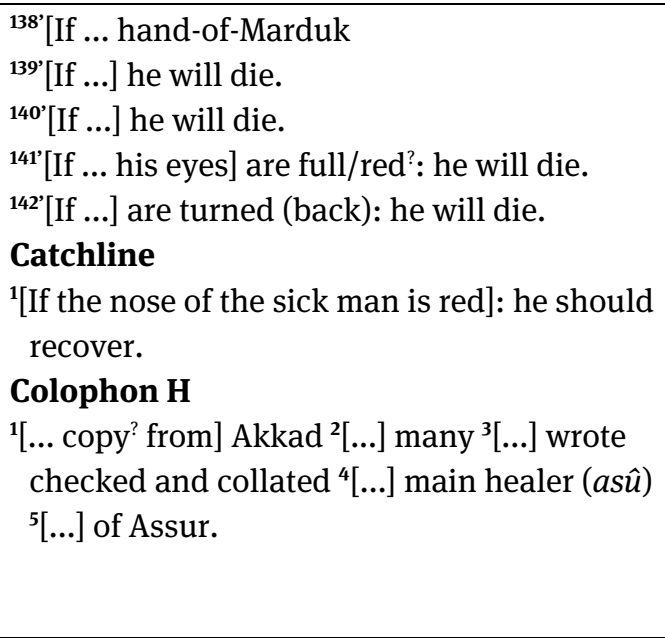 \\
\hline
\end{tabular}

\section{Transliteration}

1' A 1f.' [DIŠ] IGI `150? [...] / [(x)] LÚ 'BI? [...]

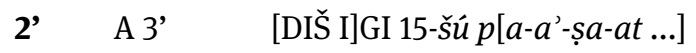

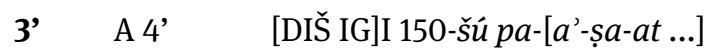

4' A 5, [DIŠ I]GI.MIN-šú pa- $a^{\prime}$-[șa-ma ...]

5' A 6' [DIŠ] IGI 15-šú pa-a'-șa- $a t^{7}-[m a$...]

6' A 7' DIŠ IGI 150-šú pa-a'-șa-at-ma [...]

7’ A 8' DIŠ IGI.MIN-šú pa-a'-ṣa-ma IM.G[Á.LI Š ŠUB.MEŠ ${ }^{\prime}$...]

8' A 9' DIŠ IGI 15-šú IM.GÁ.LI ŠUB-át [...]

9' A 10’ DIŠ IGI 150-šú IM.GÂ.LI ŠUB-át [...]

10’ A 11’ DIŠ IGI.MIN-šú IM.GÁ.LI ŠUB.MEŠ [...]

11' A 12’ DIŠ IGI 15-šú tar-kàt [...]

12’ A 13’ DIŠ IGI 150-šú tar-kàt GÍD-ma [...] 
13' A 14’ DIŠ IGI.MIN-šú tar-ka GÍD-ma [...]

14' A 15’ 'DIŠ IGI 15?-šú tar-kàt-ma NU BAD qu-b[u-ra? ...]

15' A 16’ [DIŠ IGI 150-šú tar]-kàt-ma NU BAD [...]

16’ A 17’ [DIŠ IGI.MIN-šú tar-ka-ma N]U' 'BAD? [...]

\section{BREAK OF UNKNOWN LENGTH?}

17' B i 1' ] ${ }^{\mathrm{TIN}}{ }^{2}$

18' B i 2' .] ${ }^{\top}{ }^{\urcorner}$

19' B i 3'

[ ] 'TIN??

20' B i 4' .. ${ }^{\ulcorner} \mathrm{TIN}^{\urcorner}$

21' B i 5 ' ŠU DING]IR?-`šú??

22’ B i 6 ' 'TIN'

23' B i 7 ' ŠU] 'DINGIR? URU-šú

24' B i 8' ] 'TIN?

25’ B i 9' 'DUGUD?`

26’ B i 10’ ] ${ }^{\mathrm{DUGUD}}$ ??

27’ B i 11' .] ${ }^{\ulcorner}{ }^{\urcorner}$GAM

28' B i 12' .] ${ }^{\ulcorner} \mathrm{GID}{ }^{?}-m a^{\urcorner}{ }^{r} \mathrm{TIN}^{\urcorner}$

29' B i 13' GÍ]D??-ma?? rTIN??

30' B i 14' ] ${ }^{\mathrm{GAM}}{ }^{?\urcorner}$

31' B i 15' .] ${ }^{\top}{ }^{\urcorner}[x(x)]$ 


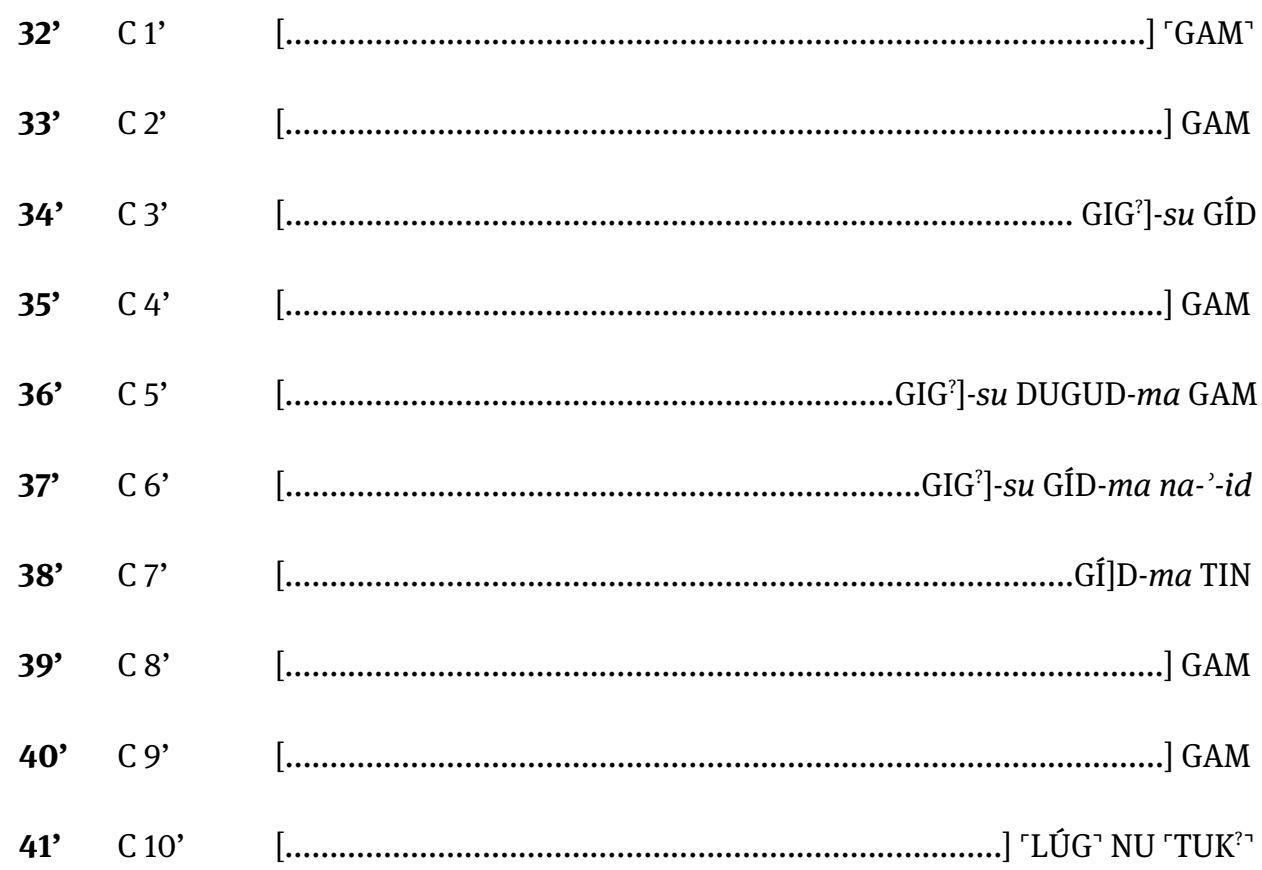

\section{BREAK OF UNKNOWN LENGTH?}

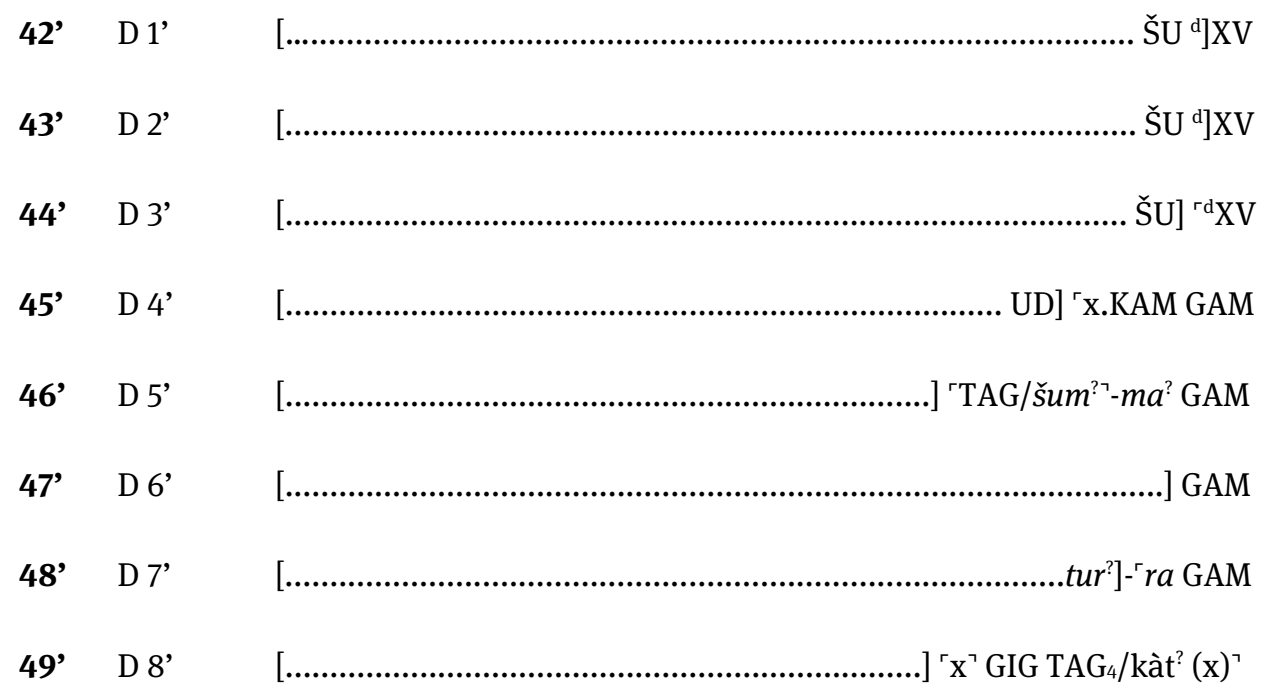

\section{BREAK OF UNKNOWN LENGTH?}

50' E 1'

' $\mathrm{X} \mathrm{X} \mathrm{X}^{\urcorner}[$ 
51' E 2' ŠU] $\mathrm{NAM}^{?\urcorner} \cdot[$ ÉRIM? $(\mathrm{x})]$

52' E 3' [DIŠ IGI 15-šú i]-la-wi u i-su-šú nu-`uš ${ }^{\top}[(\mathrm{x})]^{\ulcorner T I N}{ }^{?\urcorner}$

53’ E 4’ [DIŠ IGI 150-šú KI].MIN GIG-su DUGUD-ma TIN

54' E 5' [DIŠ IGI-šú šá 150? kap-ṣa-at? u i-s]a-šú nu?-uš-šá ŠU XXX

55' E 6ff.' [DIŠ ... IGI 1]50-šú kap-șa-at pu-us-su / [... NUN]DUM-su šá 15 na-šallu?-lu / [...] 'x šá 15 IM ina și-im-ri-šú / [...na]-־̌̌sa?-a NU ZU-e / [... Ú]H̆-su ina ŠUB-e / [...] ana EGIR SİG-iṣ

56' E 12ff.' [.......................................................] ' lu šu-ru-u'-šú / [... šá 1]50 i-šag-gúm / [...............................] x-šú šá 15 / [...] x x ${ }^{\urcorner}$

B ii 1ff.' ' 'DIŠ IGI 15?- -[šú ...] / ' 'ú-suk-ka x (x) ' [............................] / šá $150 i$-šag-

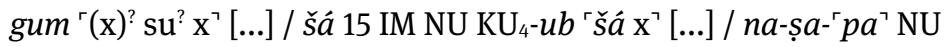

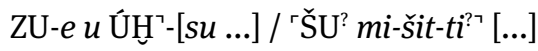

57’ B ii 7f.' DIŠ IGI 150-šú na-pal-kát-ma IGI '15?ㄱ-[šú kap-ṣa-at . / (empty) '15?ㄱ [u 150 GABA.RI SİG-iṣ?]

F 1 f. $\quad$ [...................... ]- pal? -kàt-ma IGI 15-šú kap-ṣa-at / [...] 'mur? -din-nu ú?$q \hat{\imath} / q u u^{?} 15$ u 150 GABA.RI SİG-iș

58' B ii 9f.' DIŠ IGI 15-šú GIG-ma ka-la-a-`at [ GEŠTU šá 150 MÚD 'ú'-k[al ...]

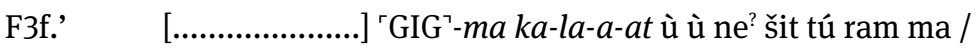
[.................... 1]50 MÚD ú-kal u ma?-diš? ana KI-nu im-qut? i-gaš? GAM

59' B ii 11' DIŠ IGI 150-šú GIG-ma 'ka-la'-a[t ...]

F 5' [...................... $]^{\ulcorner} \mathrm{GIG}^{\urcorner}-$ma ka-la-a ${ }^{?}$-at u la ina-aš-ši GAM

60’ B ii 12’ DIŠ IGI 15-šú SİG-át-ma DUL-' $m a^{\urcorner}[. .$.

F 6' [.... Sİ]G-át-ma DUL-ma NU BAD ŠU dAMAR.UTU ana UD 3.KÁM GAM

61' B ii 13’ DIŠ IGI 150-šú SİG-ât-ma DU[L ...]

F 7, [........... Sİ]G-át-ma DUL-ma NU BAD ŠU dIŠKUR ana UD 4.KÁM GAM

62' B ii 14’ 'DIŠ IGI 150?-šú SİG-ât-ma ka-l[a-a-at ...]

F 8 ، $\quad$ [................................. á] t-ma ka-la-a-at te-a-at u la ina-țal GAM

63' B ii 15' [DIŠ IGI] '150?-šú ' kát-mat [...]

F 9' .]- $m a t^{\urcorner} \mathrm{GIID}-m a \mathrm{G}\left[\mathrm{AM}^{?}\right]$ 
64' B ii 16، [DIŠ IGI 15/150?]-'šú kát-mat-ma [...]

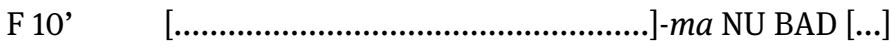

65’ B ii 17' $[\mathrm{xx}(\mathrm{x})]^{\circ} \mathrm{x} m a^{?\urcorner}[\ldots]$

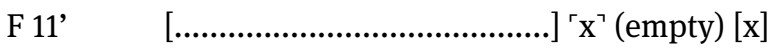

66' F 12' [.................................... (empty) ŠU DINGIR [x (x)]

67’ F 13' [.................................... TUK-šs ŠU DINGIR [x (x)]

68’ F 14f.' [...] 'x ${ }^{\ulcorner}{ }^{\urcorner}$TAG ${ }^{?}$.ME-šú ZÙ.GU[Z? x(x)] / [... ME]Š-šú ŠU DINGIR [x]

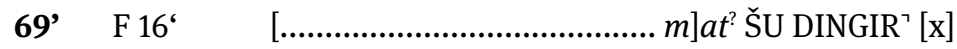

\section{BREAK OF UNKNOWN LENGTH!}

70’ C ii 1’'

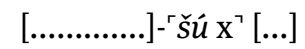

71’ C ii 2’ DIŠ IGI.MIN-šú 'IM?'.[GÚ ...]

72’ C ii 3’ DIŠ IGI.MIN-šú IM.G[Ú ...]

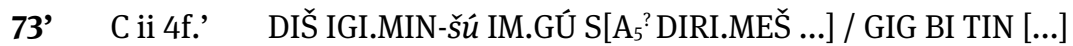

74’ C ii 6’ DIŠ IGI.MIN-šú MÚD DIRI.[MEŠ ...]

75’ C ii 7’ DIŠ IGI.MIN-šú MÚD DIRI.MEŠ x[...]

76’ C ii 8’ DIŠ IGI.MIN-šú MÚD DIRI.MEŠ x[...]

77’ C ii 9f.' DIŠ IGI.MIN-šú MÚD DIRI.MEŠ [...] / IM.RI. ' ${ }^{?\urcorner\urcorner}[. .$.

78’ C ii 11’ DIŠ IGI.MIN-šú [...]

\section{BREAK OF UNKNOWN LENGTH!}

79, D ii 1 ' $\quad$ 'DIŠ $[\ldots]$

G 1' [DIŠ IGI.MIN]-ršú x? [...]

80’ D ii 2' DIŠ IGI. 'MIN'-[šú ...]

G 2' [DI]Š IGI.MIN-šú 'GEN ${ }_{7}^{\text {? }} \mathrm{x}^{\urcorner}{ }^{\mathrm{r}}[\ldots]$ 

81' D ii 3'
DIŠ IGI.MIN-šú [...]
G 3' [DI] Š IGI.MIN-šú GEN ${ }_{7}^{?}{ }^{?}$ ri $/ \mathrm{sag}^{? ?}$-[...]
82' D ii 4' DIŠ IGI.MIN-šú Î[R ...]
G 4' [DIŠ I]GI.MIN-šú ÍR-a ${ }^{\ulcorner} \mathrm{GE}_{6}{ }^{7}[\ldots]$
83’ D ii 5' DIŠ IGI.MIN-šú Í[R ...]
G 5' [DIŠ IGI].'MIN '-šú ÎR-a bir-d[u ...]
84' D ii 6' DIŠ 'IGI'.[MIN-šú ...]
G 6' [DIŠ IGI.MIN-šú] 'ÍR TUK.T[UK- $a^{?}$...]
85’ G 7’ [DIŠ IGI.MIN-šú Í]R ŠUB.ŠUB- $\left[a^{3}\right.$...]

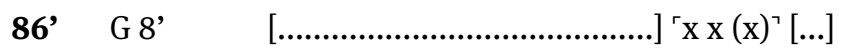

\section{BREAK OF UNKNOWN LENGTH!}

87’ $\quad$ E ii 1' $\quad$ 'DIŠ IGI.MIN?'-[̌̌ú ...]

88' E ii 2f.' DIŠ IGI.MIN- 'šû’ [...] / GIG 'B[I/-su? ...]

89’ E ii 4’ DIŠ IGI.MIN-šú bal-[șa-ma? ...]

90’ E ii 5’ DIŠ IGI.MIN-šú bal-[șa-ma? ...]

91' E ii 6, DIŠ IGI.MIN-šú na-bal-[ku-ta-ma? ...]

92’ E ii 7’ DIŠ IGI.MIN-šú na-bal-[ku-ta-ma? ...]

93’ E ii 8’ DIŠ IGI.MIN-šú ŠUB-tas ina $^{3}$ x [...]

94' E ii 9’ DIŠ IGI.MIN-šú ŠUB-ma [...]

95’ E ii 10’ DIŠ IGI.MIN-šú ŠUB-ma [...]

96' E ii 11’ DIŠ IGI.MIN-šú ŠUB-ma [...]

97’ E ii 12’ DIŠ IGI.MIN-šú ŠUB-ma [...]

98' E ii 13f.' DIŠ IGI.MIN-šú ŠUB-ma [...] / 'x x x [...] 


\section{BREAK OF UNKNOWN LENGTH!}

99' H iii 1’ [DIŠ] 'IGI'.[MIN-šú ...]

100’ H iii 2ff.' DIŠ IGI.MIN-[šú ...] / TA [GIG-su it-tab-šú? ana A.GA.NU.TIL-le-e] / GUR-šu[m-ma? ina UD BI.IZ AN-e? GAM?]

101' H iii 5' 'DIŠ` [IGI.MIN]-`šú it-te?-[...]

102’ H iii 6’ DIŠ IGI.MIN-[šú i]t-te-'né-[...]

103’ H iii 7’ DIŠ IGI.MIN-šú TA[G? ...]

104’ H iii 8f' $\quad$ DIŠ IGI.MIN-šú iṣ-ṣa-nun- $d\left[a^{\text {? .... }] / ~ u ~ U B . N I G I N . N A . B I ~ ' D U ? ? ~ .[M E S ̌ ? ~ . . .] ~}\right.$

105’ H iii 10’ DIŠ IGI.MIN-šú iṣ-șa-nun-da u [...]

106’ H iii 11' DIŠ IGI.MIN-šú iz-za-ka ni/q[á? ...]

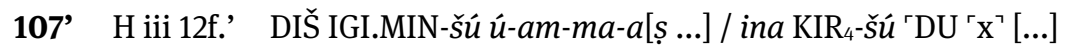

108' H iii 14’ DIŠ IGI.MIN-šú bé-e-[ra ...]

109’ H iii 15’ DIŠ IGI.MIN-šú DUGUD-ma [...]

110’ H iii 16’ DIŠ IGI.MIN-šú UG[U ...]

111’ H iii 17’ DIŠ IGI.MIN-šú tar-[...]

112’ H iii 18’ DIŠ IGI.MIN-šú ' $x^{\urcorner}[\ldots]$

\section{BREAK OF UNKNOWN LENGTH!}

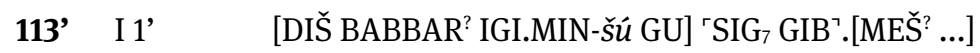

114' I ' [DIŠ BABBAR? IGI.MIN-šú] SIG $_{7} b a-l i l^{?\urcorner}[\ldots]$

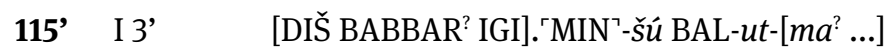

116' I 4' [DIŠ BABBAR? IGI].MIN-šú BAL-ut-ma AN.TA $u$ [KI.TA ...]

117’ I 5’ DIŠ BABBAR IGI.MIN-šú BAL-ut-ma GÌR.MIN-šú SED.[MEŠ? ...] 


\begin{tabular}{|c|c|c|}
\hline 118' & I 6 & DIŠ BABBAR IGI.MIN-šú BAL-ut-ma GÌR.MIN-šú ZI ' x ${ }^{\urcorner}[\ldots]$ \\
\hline 119 & I 7 & DIŠ GE 6 IGI.MIN-šú BAL-ut-[ma? ...] \\
\hline 120 ' & I 8 & DIŠ GE 6 IGI.MIN-šú BAL-ut-ma GÌR.MIN-šú ana ŠÀ?-[šú tur-ra? ...] \\
\hline 121 & I 9f.' & DIŠ SA IGI.MIN-šú GEN ${ }_{7}$ ŠU.GUR?(SI) kap-pu [...] / na-kid [...] \\
\hline 122 & I 11f'. & $\begin{array}{l}\text { DIŠ SA IGI.MIN-šú GEN } \text { Ǧi } \text {-te-e [...] / ŠU DINGIR-šú lu ŠU DINGIR } \\
\text { UR[U-šú ...] }\end{array}$ \\
\hline
\end{tabular}

123’ I 13’ DIŠ SA IGI.MIN-šú DU.MEŠ-ma šá SAG.KI GAR- ${ }^{\prime} n u^{\urcorner}[\ldots]$
124’ I 14’ DIŠ SA kak-kul-ti IGI 15-šú GE.MEŠ [...]
125’ I 15’ DIŠ SA kak-kul-ti IGI 150-šú GE.MEŠ [...]
126’ I 16’ DIŠ SA kak-kul-ti IGI.MIN-šú GE.MEŠ [...]
127’ I 17’ DIŠ SA kak-kul-ti IGI 15-šú tar-ku [...]
128' I 18' DIŠ SA kak-kul-ti IGI 150-šú tar-ku [...]
129’ I 19' DIŠ SA kak-kul-ti IGI 15-šú ' $d\left[u^{\prime}-(\hat{u})-m u\right.$... $]$
130’ I 20’ DIŠ SA kak-kul-ti IGI 150-šú [...]
131’ I 21’ DIŠ SA kak-kul-ti IGI.MIN-šú [...]
132’ I 22f.' DIŠ PIŠ 10 IGI.MIN-šú MÚ.ME[Š-ha ...] / MÚ.MEŠ-h̆u 'x x [...]
J 1' [...] ' ${ }^{\prime}{ }^{\urcorner}[\ldots]$
133’ I 24' DIŠ PA IGI.MIN-šú 2.T[A ... ]

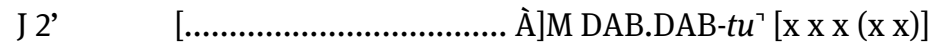
134' I 25f.' [DIŠ P]A IGI.MIN-šú šá 1[5 .......................................] / ŠU d [...]

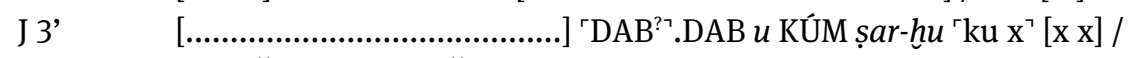 $\left[\ldots . . . \mathrm{S}^{\mathrm{d}}{ }^{\mathrm{r}}{ }^{r} \mathrm{XXX} \mathrm{X}^{?\urcorner}: \check{S ̆ U}^{\mathrm{d} r} \mathrm{UTU}^{?\urcorner}\right.$

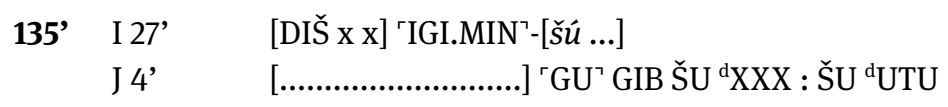




\begin{tabular}{|c|c|c|}
\hline $136^{\prime}$ & J 5 & [DIŠ ... S]A ${ }^{?}$ SIG $_{7}$ GIB ŠU d XXX :? ŠU d UTU \\
\hline 137 & J 6', & $\left.[\ldots]^{\ulcorner} \mathrm{x}^{\urcorner} \check{S}^{\mathrm{d}}{ }^{\mathrm{d}} \mathrm{MAS}\right\urcorner$ \\
\hline 138' & $\mathrm{J} 7$ & {$[\ldots]^{\ulcorner} \mathrm{KIMIN}^{?}{ }^{\mathrm{d}} \mathrm{AMAR}^{?\urcorner} . \mathrm{UTU}$} \\
\hline 139' & $\mathrm{J} 8$ & {$[\ldots]^{\ulcorner} \mathrm{x}^{\urcorner} \mathrm{GAM}$} \\
\hline $140^{\prime}$ & J 9' & [...] (empty) GAM \\
\hline $141^{\prime}$ & $\mathrm{J} 10$ & 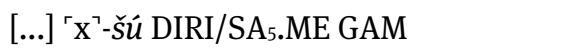 \\
\hline 142’ & J 11’' & [...] tur-ra GAM \\
\hline
\end{tabular}

\section{Catchline}

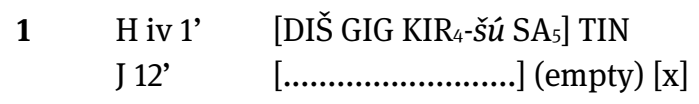

\section{Colophon H}

1 H iv 2' [... GABA.RI? kur $] a k^{?}-k a-d e-e$

$2 \quad$ H iv 3, [..m $\left.a^{?}\right]-^{\prime}-d u-u ́-t i$

$3 \quad \mathrm{H}$ iv 4' [...] šá-țir is-sa-niq 'ba ' ${ }^{\urcorner}{ }^{\ulcorner} r i$

$4 \quad \mathrm{H}$ iv 5, [... $]^{\text {lúGAL A.ZU.MEŠ }}$

$5 \quad \mathrm{H}$ iv 6, [...] aš-šur ${ }^{\mathrm{ki}}$

\section{§ Vla A Note on the Physiognomic Omens Alamdimmû tablet 8}

The passages in question are edited by B. Böck 2000: 110ff. (Lines: 61-72, 109-110), manuscripts B (82-5-22, 196a = CT 28 Pl. 28-29, Late Assyrian). Böck understands that the relevant passages are concerned with the human eyes. Nevertheless, there are strong warnings against Böck's interpretation: the syllabic spellings of IGI in lines 23, 31, 73, 91, 95 and 111 (Böck 2000: 110, 112, 114) as pa-nu/ni rather suggest that these passages are concerned with the human face and not with the eyes. Therefore, they are not re-edited here. 